\title{
Binary stars with RR Lyrae components - new candidates in the Galactic bulge
}

\author{
M. Skarka ${ }^{1,2}$, Z. Prudil ${ }^{3}$ and J. Liška ${ }^{4}$ \\ 1 Department of Theoretical Physics and Astrophysics, Masaryk University, \\ Kotlárská 2, 61137 Brno, (E-mail: maska@physics.muni.cz) \\ 2 Astronomical Institute of the Czech Academy of Sciences, Fričova 298, \\ 25165 Ondřejov, The Czech Republic Astronomical Institute of the Czech \\ Academy of Sciences \\ 25165 Ondřejov, The Czech Republic \\ 3 Astronomisches Rechen-Institut, Zentrum für Astronomie der Universität at \\ Heidelberg, Mönchhofstr. 12-14, 69120 Heidelberg, Germany \\ ${ }^{4}$ Central European Institute of Technology - Brno University of Technology \\ (CEITEC BUT), Purkyñova 656/123, CZ-61200 Brno,Czech Republic
}

Received: October 29, 2019; Accepted: February 5, 2020

\begin{abstract}
There is a significant lack of binary stars with RR Lyrae components. In this brief contribution, we introduce 20 new candidates in the Galactic bulge identified on the basis of the Light-Travel-Time Effect with expected orbital periods between 3 and 15 years.

Key words: Stars: RR Lyrae - stars: binaries - Galactic bulge
\end{abstract}

\section{Introduction}

The mass of a star can be independently determined only when it orbits a common center of mass with another body in a binary system. This is something that is sadly lacking for RR Lyrae stars. There is only one RR Lyrae type star that is known to reside in the binary system (Liška et al., 2016a), and about one hundred candidates (listed in the online database Liska \& Skarka, 2016) ${ }^{1}$ determined mostly indirectly via the Light-Travel-Time Effect (LTTE, e.g. Hajdu et al., 2015; Liška et al., 2016b) or through proper-motion anomalies (Kervella et al., 2019).

Together with Cepheids and other radially pulsating stars, RR Lyrae stars serve as classical distance standard candles, and thus, it is crucial to know their masses accurately. Unfortunately, their masses are known only roughly from evolutionary and pulsation models (e.g. Sweigart, 1987; Popielski et al., 2000). An independent and accurate mass determination might be possible if the RR Lyrae star is identified as an eclipsing binary component, which would

${ }^{1}$ https://rrlyrbincan.physics.muni.cz/ 
not be an easy task. Due to the advanced evolutionary state of the RR Lyrae star (a horizontal-branch giant) the companion would most probably have to be a degenerate stellar remnant or low-mass main-sequence star, and in both cases it would be a small, faint object producing only shallow or no eclipses. Binary systems with other horizontal-branch or red-giant star components would produce more distinct observables. Such binaries, however, are less likely to be observed due to the short life-time of these evolutionary phases.

In addition, in order to avoid mass transfer during the evolution of the system prior to the formation of the classical RR Lyrae star, only wide systems are expected to have RR Lyrae components (Karczmarek et al., 2017). Thanks to photometric observations spanning decades, sometimes even one century, the easiest way to reveal candidates is through the light-time-travel effect (LTTE) (Sterken, 2005). However, without confirmation using radial-velocity measurements, the candidates cannot be assumed to be binary stars (Skarka et al., 2018).

\section{New candidates}

In our study focused on searching for additional binary candidates via cyclic period variations (Prudil et al., 2019), we investigated over 9000 stars from the Galactic bulge. Because long-term period changes can be confused with the long-term Blazhko effect, we selected only stars without detectable modulation based on the study by Prudil \& Skarka (2017). We used data from the OGLEIII and IV phases (Soszyński et al., 2011, 2014), and the KMTNet survey (Lee et al., 2014), which give us datasets sometimes spanning more than 20 observing seasons.

For the investigation of the period changes, we used the method developed by Hertzsprung (1919). Each observing season was divided into two bins which were fitted with Fourier series using the OGLE ephemerides. The time delay was estimated from the phase shift of the light curves.

Initially, we found 200 stars with suspicious O-C's. Among these stars, the binary candidates identified by Hajdu et al. (2015) were also detected. To strenghten the reliability of our candidates, we performed the test introduced by Shibahashi (2017). We ended up with 20 strong binary candidates. Period variations of these 20 candidates were fitted assuming binarity by using expressions introduced by Irwin (1952). After fitting we get the full characterization of the orbits. The parameter ranges of the orbital parameters are listed in Table 1, the particular values and the full list can be found in Prudil et al. (2019).

\section{Summary and future prospects}

We complemented the currently known sample of RR Lyrae binary component candidates with 20 additional stars. The values are in accordance with expecta- 
Table 1. Orbital parameter ranges of our 20 candidtes.

\begin{tabular}{cccccc}
\hline \hline$P_{\text {orb }}[\mathrm{d}]$ & $a \sin (i)[\mathrm{au}]$ & $e$ & $\omega[\mathrm{deg}]$ & $K\left[\mathrm{~km} \mathrm{~s}^{-1}\right]$ & $f(M)\left[\mathrm{M}_{\odot}\right]$ \\
\hline $1180-5041$ & $0.18-4.28$ & $0.05-0.61$ & $-362-272$ & $0.87-9.82$ & $0.0002-0.412$ \\
\hline \hline
\end{tabular}

tions given by the used data and are similar to those published by Hajdu et al. (2015). Three of our candidate stars have assumed orbital periods less than 10 years and rather large amplitudes. Assumed companions of two of these stars are supposed to have minimal masses comparable or larger than the RR Lyrae component. In some circumstances (horizontal or asymptotic-giant branch nature of the companion) possible eclipses could be observed. In any case, all our candidate stars must be confirmed by radial-velocity observations, which will be the natural next step. Untill the candidates are confirmed using radial velocity observations, all the companions, periods, orbital parameters, etc. are only suspected.

Acknowledgements. MS acknowledges the OP VVV project Postdoc@MUNI No. CZ.02.2.69/0.0/0.0/16-027/0008360. ZP acknowledges the support of the Hector Fellow Academy. JL acknowledges support of the project CEITEC 2020 (LQ1601) with financial support from the Ministry of Education, Youth and Sports of the Czech Republic under the National Sustainability Programme II.

\section{References}

Hajdu, G., Catelan, M., Jurcsik, J., et al., New RR Lyrae variables in binary systems. 2015, Mon. Not. R. Astron. Soc., 449, L113, DOI: 10.1093/mnrasl/slv024

Hertzsprung, E., Bearbeitung der J.F.J. Schmidtschen Beobachtungen und Bestimmung der Periode von $\delta$ Cephei. 1919, Astronomische Nachrichten, 210, 17, DOI: 10.1002/asna.19202100202

Irwin, J. B., The Determination of a Light-Time Orbit. 1952, ApJ, 116, 211, DOI: $10.1086 / 145604$

Karczmarek, P., Wiktorowicz, G., Iłkiewicz, K., et al., The occurrence of binary evolution pulsators in classical instability strip of RR Lyrae and Cepheid variables. 2017, Mon. Not. R. Astron. Soc., 466, 2842, DOI: 10.1093/mnras/stw3286

Kervella, P., Gallenne, A., Remage Evans, N., et al., Multiplicity of Galactic Cepheids and RR Lyrae stars from Gaia DR2. I. Binarity from proper motion anomaly. 2019, Astron. Astrophys., 623, A116, DOI: 10.1051/0004-6361/201834210

Lee, C.-U., Kim, S.-L., Cha, S.-M., et al., Observational performance of the KMTNet. 2014, in Society of Photo-Optical Instrumentation Engineers (SPIE) Conference Series, Vol. 9145, Proc. SPIE, $91453 \mathrm{~T}$ 
Liska, J. \& Skarka, M., Database of candidates for RR Lyrae stars in binary systems RRLyrBinCan. 2016, Commmunications of the Konkoly Observatory Hungary, 105, 209

Liška, J., Skarka, M., Mikulášek, Z., Zejda, M., \& Chrastina, M., New analysis of the light time effect in TU Ursae Majoris. 2016a, Astron. Astrophys., 589, A94, DOI: 10.1051/0004-6361/201525870

Liška, J., Skarka, M., Zejda, M., Mikulášek, Z., \& de Villiers, S. N., Cyclic variations in O-C diagrams of field RR Lyrae stars as a result of LiTE. 2016b, Mon. Not. R. Astron. Soc., 459, 4360, DOI: 10.1093/mnras/stw851

Popielski, B. L., Dziembowski, W. A., \& Cassisi, S., Petersen Diagram for RRd Stars in the Magellanic Clouds. 2000, AcA, 50, 491

Prudil, Z. \& Skarka, M., Blazhko effect in the Galactic bulge fundamental mode RR Lyrae stars - I. Incidence rate and differences between modulated and nonmodulated stars. 2017, Mon. Not. R. Astron. Soc., 466, 2602, DOI: 10.1093/mnras/stw3231

Prudil, Z., Skarka, M., Liška, J., Grebel, E. K., \& Lee, C. U., Candidates for RR Lyrae in binary systems from the OGLE Galactic bulge survey. 2019, Mon. Not. R. Astron. Soc., 487, L1, DOI: 10.1093/mnrasl/slz069

Shibahashi, H., The Blazhko RR Lyrae variables and phase modulation in binary systems. 2017, in European Physical Journal Web of Conferences, Vol. 152, European Physical Journal Web of Conferences, 03006

Skarka, M., Liška, J., Dřevěný, R., et al., A cautionary tale of interpreting O-C diagrams: period instability in a classical RR Lyr Star Z CVn mimicking as a distant companion. 2018, Mon. Not. R. Astron. Soc., 474, 824, DOI: 10.1093/mnras/stx2737

Soszyński, I., Dziembowski, W. A., Udalski, A., et al., The Optical Gravitational Lensing Experiment. The OGLE-III Catalog of Variable Stars. XI. RR Lyrae Stars in the Galactic Bulge. 2011, AcA, 61, 1

Soszyński, I., Udalski, A., Szymański, M. K., et al., Over 38000 RR Lyrae Stars in the OGLE Galactic Bulge Fields. 2014, AcA, 64, 177

Sterken, C., The O-C Diagram: Basic Procedures. 2005, in Astronomical Society of the Pacific Conference Series, Vol. 335, The Light-Time Effect in Astrophysics: Causes and cures of the $O-C$ diagram, ed. C. Sterken, 3

Sweigart, A. V., Evolutionary Sequences for Horizontal-Branch Stars. 1987, Astrophys. J., Suppl., 65, 95, DOI: 10.1086/191219 